Supplementary Material for

\title{
Synthesis of Tetraphenylporphyrinate Manganese(III) Siloxides by Silyl Group Transfer from Silanethiols
}

\author{
Daniel J. Meininger, Zeiph Kasrawi, Hadi D. Arman, and Zachary J. Tonzetich* \\ Department of Chemistry, University of Texas at San Antonio, San Antonio, TX, USA \\ zachary.tonzetich@utsa.edu
}

Contents

Pages

Figure S1. ${ }^{1} \mathrm{H}$ NMR spectrum of $\left[\mathrm{Mn}\left(\mathrm{OSi}^{i} \mathrm{Pr}_{3}\right)(\mathrm{TPP})\right]$.

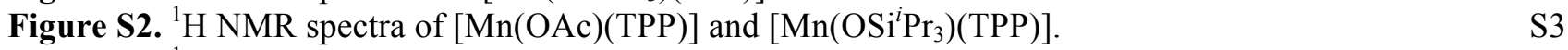

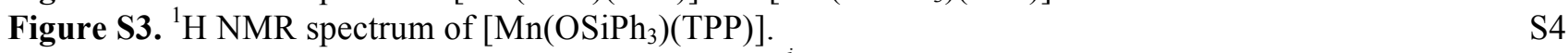

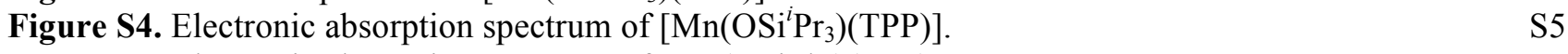

Figure S5. Electronic absorption spectrum of $\left[\mathrm{Mn}\left(\mathrm{OSiPh}_{3}\right)(\mathrm{TPP})\right] . \quad \mathrm{S} 6$

$\begin{array}{lr}\text { Figure S6. HRMS of }\left[\mathrm{Mn}\left(\mathrm{OSi}^{i} \mathrm{Pr}_{3}\right)(\mathrm{TPP})\right] . & \mathrm{S} 7\end{array}$

$\begin{array}{lr}\text { Figure S7. HRMS of }\left[\mathrm{Mn}\left(\mathrm{OSiPh}_{3}\right)(\mathrm{TPP})\right] . & \mathrm{S} 8\end{array}$

$\begin{array}{lr}\text { Table S1. Crystallographic data and refinement parameters. } & \text { S9 }\end{array}$ 


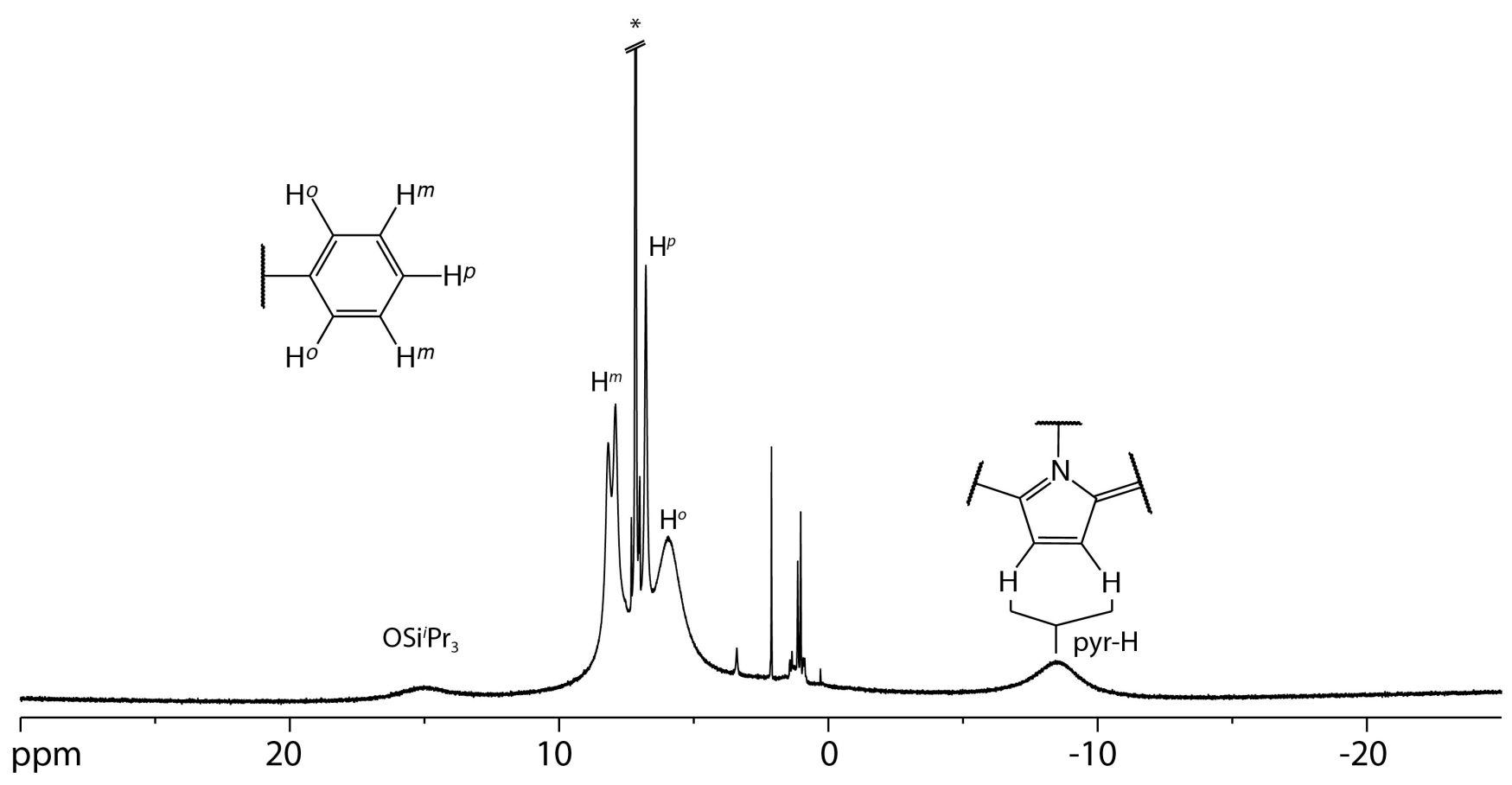

Figure S1. $500 \mathrm{MHz}{ }^{1} \mathrm{H}$ NMR spectrum of $\left[\mathrm{Mn}\left(\mathrm{OSi}^{i} \mathrm{Pr}_{3}\right)(\mathrm{TPP})\right]$ in benzene- $\mathrm{d}_{6}$ showing peak assignments. Asterisk denotes resonance due to $\mathrm{C}_{6} \mathrm{D}_{5} \mathrm{H}$. The peaks between 0 and $5 \mathrm{ppm}$ include resonances attributable to toluene and pentane from recrystallization. 


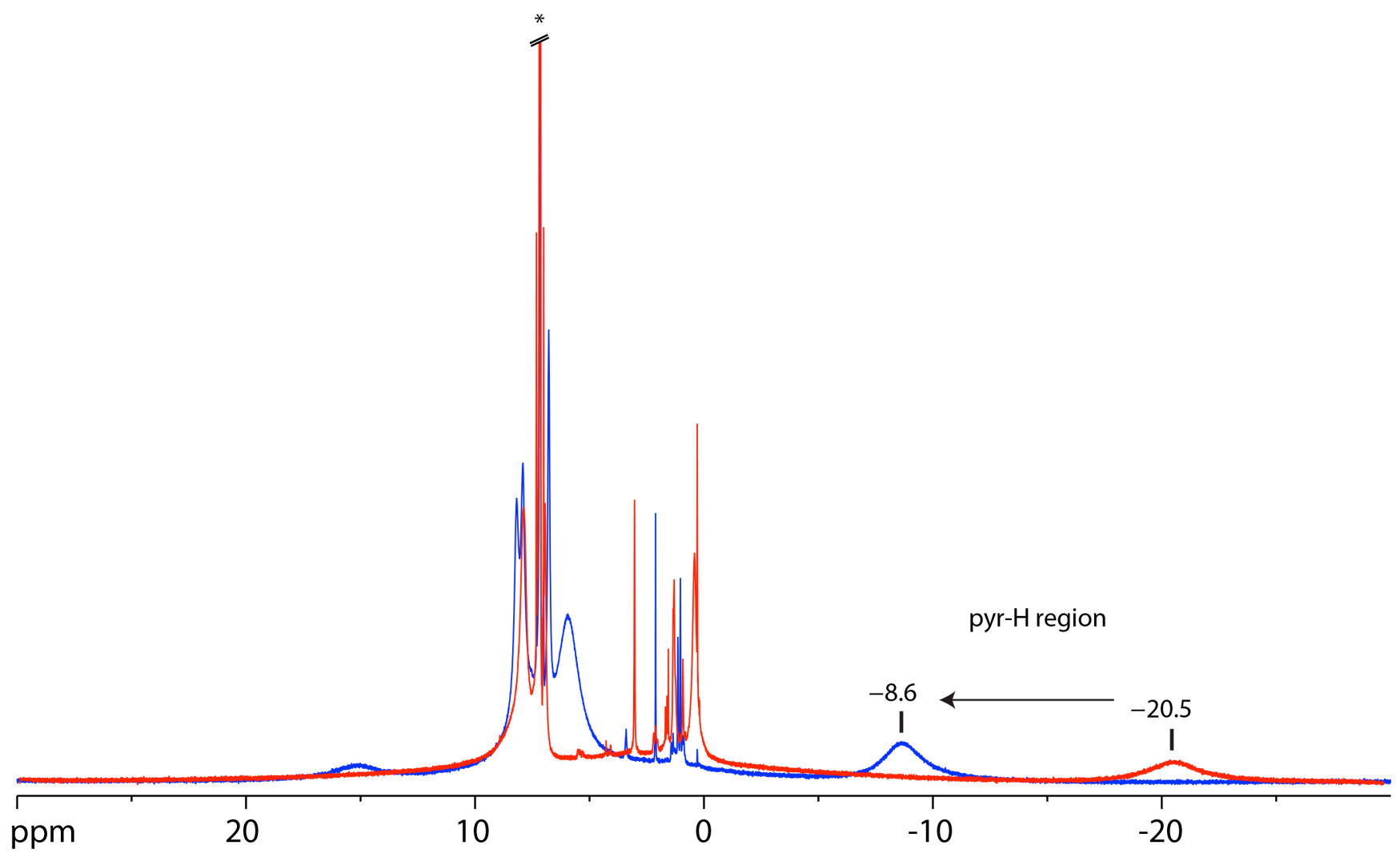

Figure S2. Overlay of the $500 \mathrm{MHz}{ }^{1} \mathrm{H}$ NMR spectra of $[\mathrm{Mn}(\mathrm{OAc})(\mathrm{TPP})]$ (red) and $\left[\mathrm{Mn}\left(\mathrm{OSi}^{i} \mathrm{Pr}_{3}\right)(\mathrm{TPP})\right]$ (blue) in benzene- $\mathrm{d}_{6}$ showing the upfield shift of the pyrrolic resonance upon substitution of the acetate group for the siloxide. 


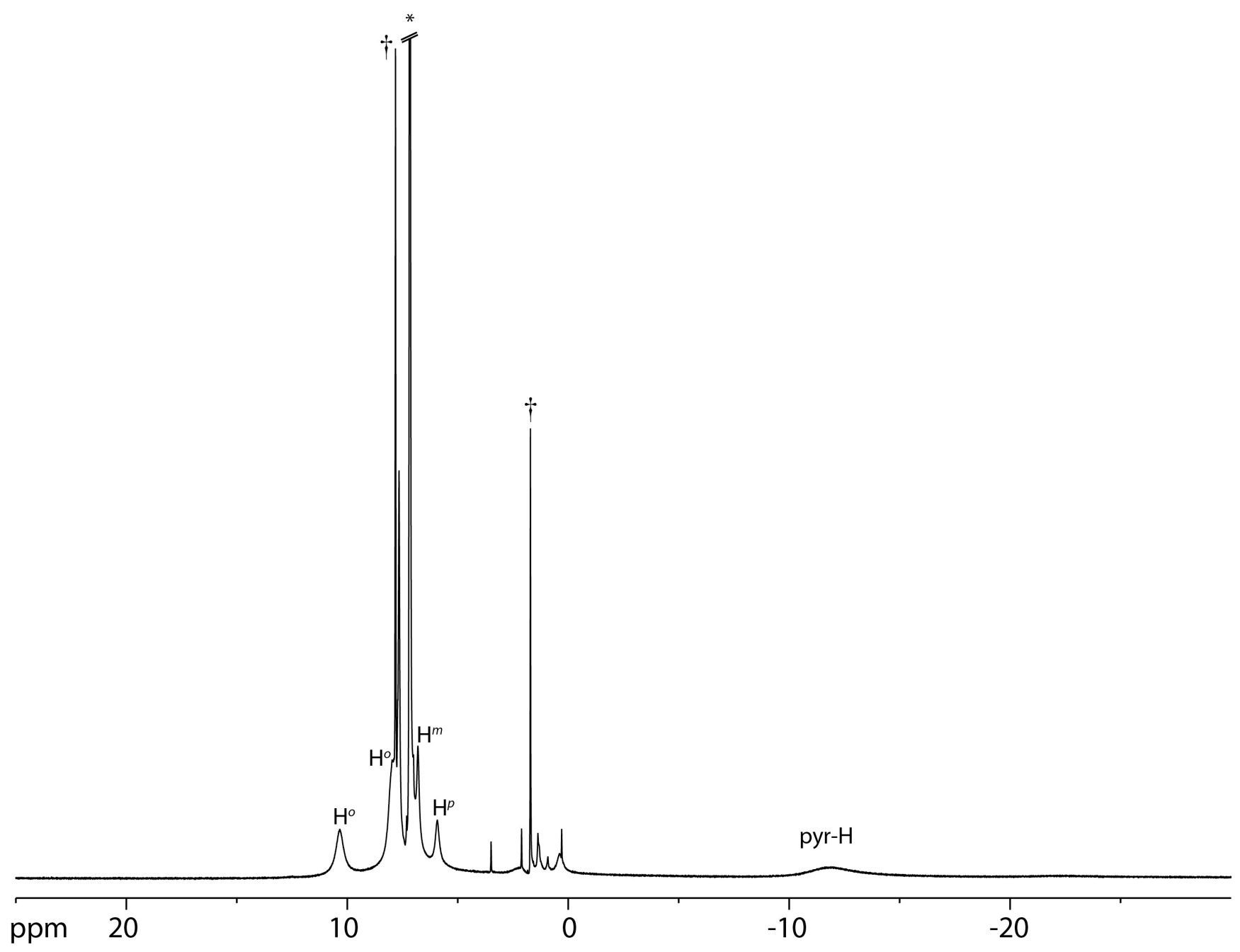

Figure S3. $500 \mathrm{MHz}{ }^{1} \mathrm{H}$ NMR spectrum of $\left[\mathrm{Mn}\left(\mathrm{OSiPh}_{3}\right)(\mathrm{TPP})\right]$ in benzene- $\mathrm{d}_{6}$ showing peak assignments. Asterisk denotes resonance due to $\mathrm{C}_{6} \mathrm{D}_{5} \mathrm{H}$. The cross denotes peaks due to excess $\mathrm{HSSiPh}_{3}$. 


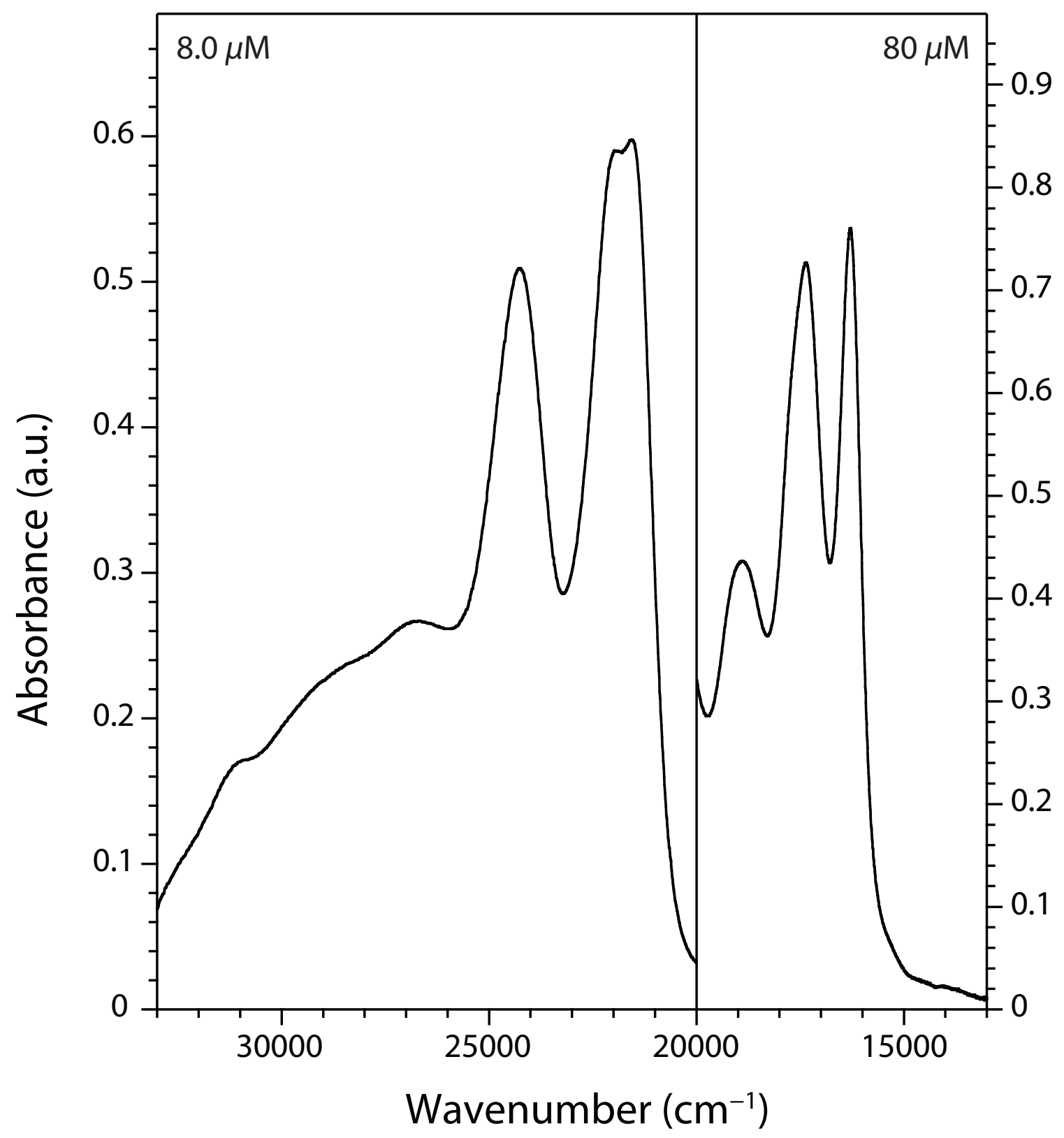

Figure S4. Electronic absorption spectrum of $\left[\mathrm{Mn}\left(\mathrm{OSi}^{i} \mathrm{Pr}_{3}\right)(\mathrm{TPP})\right]$ in toluene. 


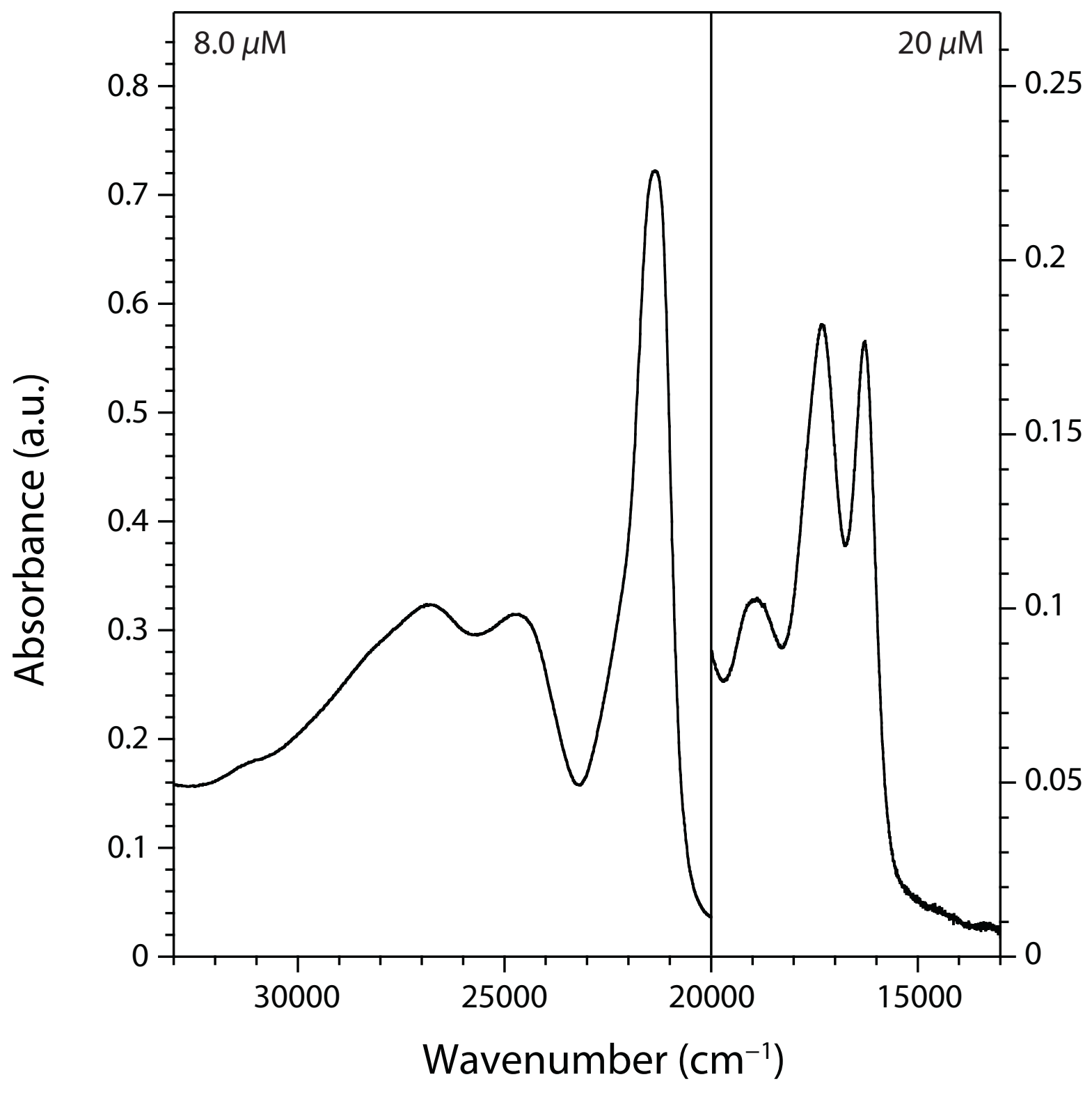

Figure S5. Electronic absorption spectrum of $\left[\mathrm{Mn}\left(\mathrm{OSiPh}_{3}\right)(\mathrm{TPP})\right]$ in toluene. 


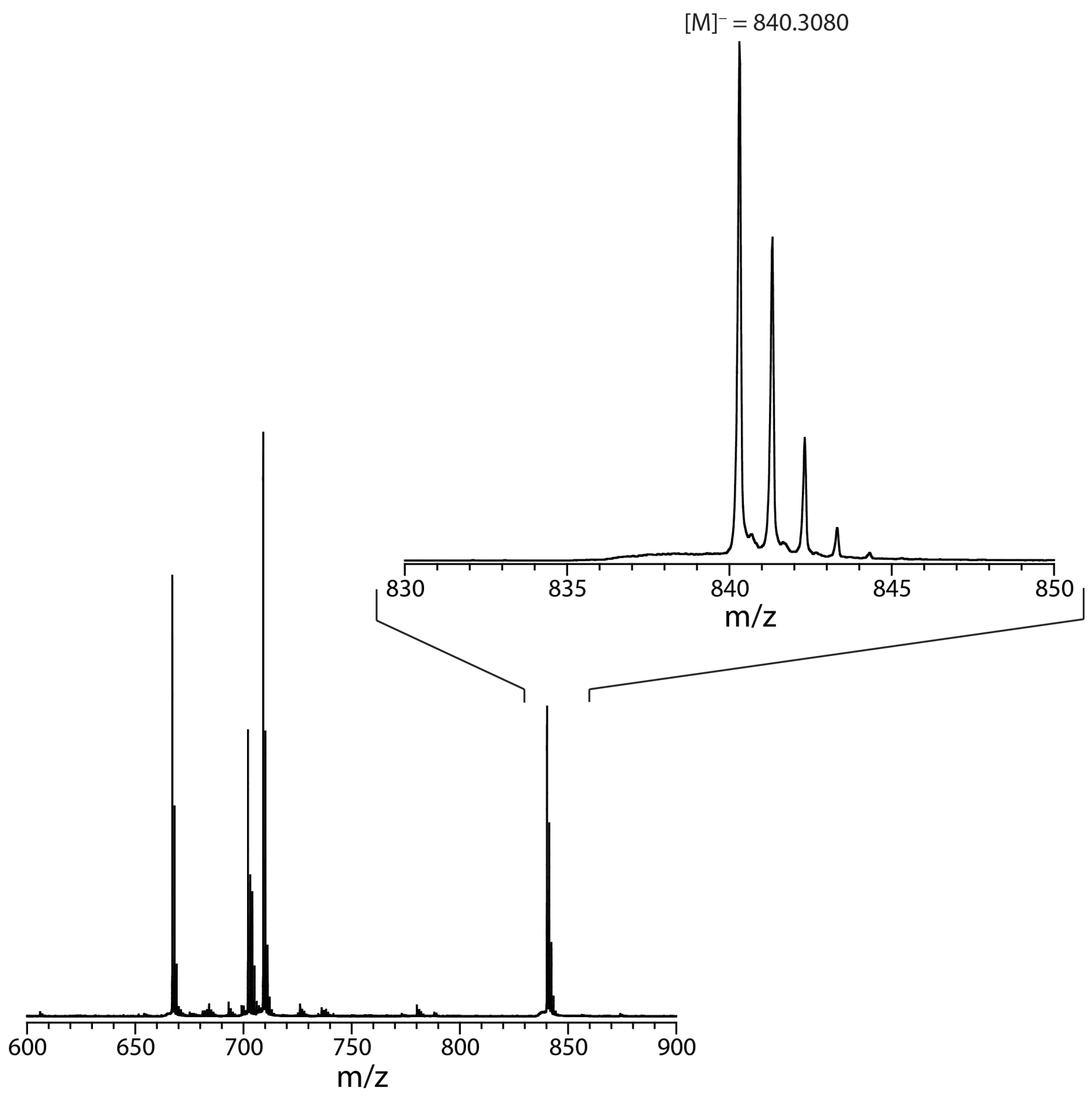

Figure S6. HRMS (APCI, negative mode) of $\left[\mathrm{Mn}\left(\mathrm{OSi}^{i} \mathrm{Pr}_{3}\right)(\mathrm{TPP})\right]$. 


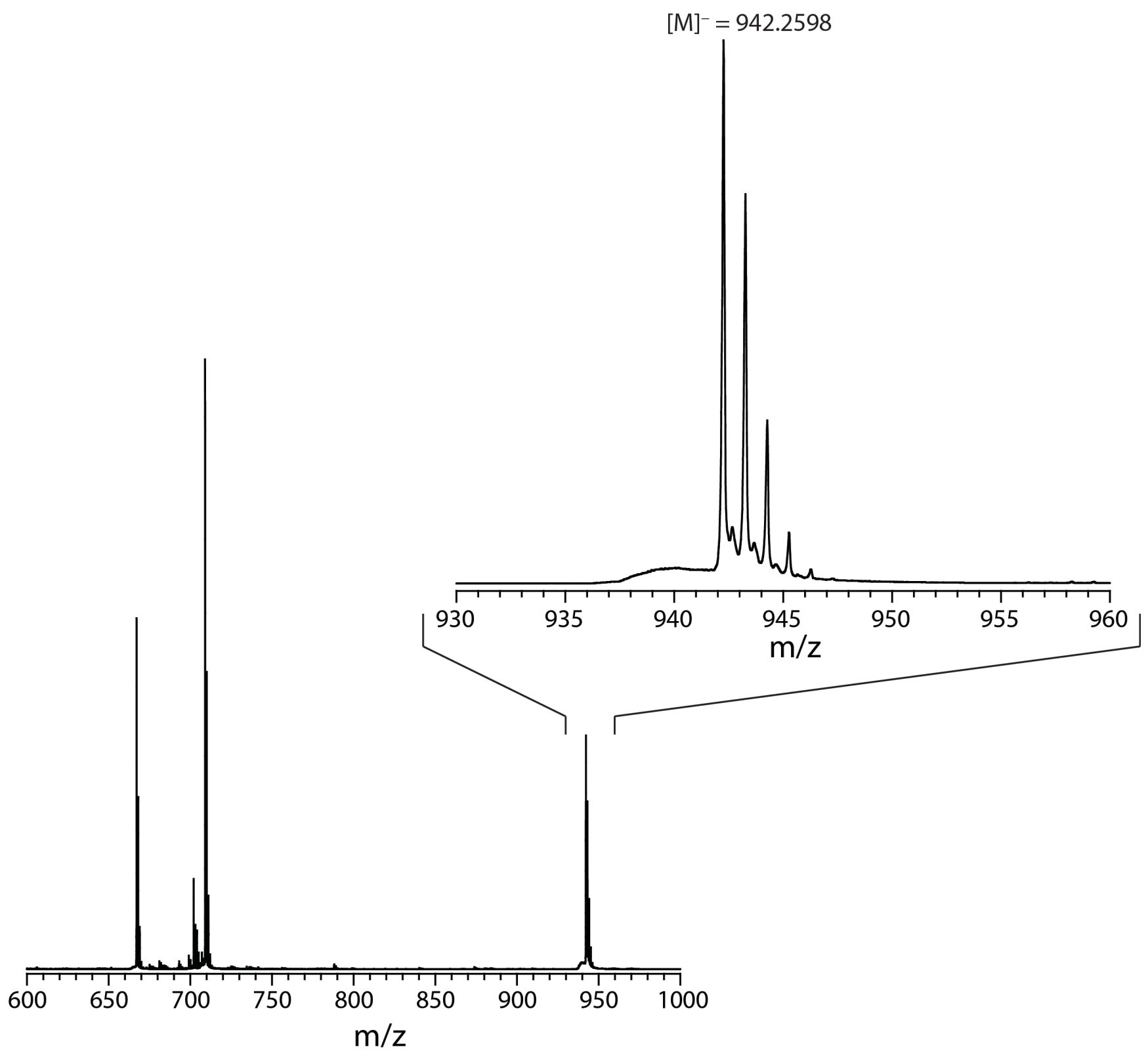

Figure S7. HRMS (APCI, negative mode) of $\left[\mathrm{Mn}\left(\mathrm{OSiPh}_{3}\right)(\mathrm{TPP})\right]$. 
Table S1. Crystallographic data and refinement parameters for $\left[\mathrm{Mn}\left(\mathrm{OSi}^{i} \mathrm{Pr}_{3}\right)(\mathrm{TPP})\right]$ and $\left[\mathrm{Mn}\left(\mathrm{OSiPh}_{3}\right)(\mathrm{TPP})\right]$. $^{\S}$

\begin{tabular}{|c|c|c|}
\hline Compound & {$\left[\mathrm{Mn}\left(\mathrm{OSi}^{i} \mathrm{Pr}_{3}\right)(\mathrm{TPP})\right]$} & {$\left[\mathrm{Mn}\left(\mathrm{OSiPh}_{3}\right)(\mathrm{TPP})\right]$} \\
\hline Empirical formula & $\mathrm{C}_{53} \mathrm{H}_{49} \mathrm{MnN}_{4} \mathrm{OSi}$ & $\mathrm{C}_{62} \mathrm{H}_{43} \mathrm{MnN}_{4} \mathrm{OSi}$ \\
\hline Formula weight $(\mathrm{g} / \mathrm{mol})$ & 840.99 & 943.03 \\
\hline Temperature $(\mathrm{K})$ & $98(2)$ & 293(2) \\
\hline Crystal system, space group & Triclinic, $P \overline{1}$ & Triclinic, $P \overline{1}$ \\
\hline \multirow{2}{*}{ Unit cell dimensions $(\AA)$} & $\begin{array}{l}a=12.482(3) \\
b=13.124(4) \\
c=14.834(3)\end{array}$ & $\begin{array}{l}a=11.953(5) \\
b=13.955(6) \\
c=16.102(8)\end{array}$ \\
\hline & $\begin{array}{l}\alpha=75.206(17) \\
\beta=65.422(13) \\
\gamma=85.016(16)\end{array}$ & $\begin{array}{l}\alpha=101.262(8) \\
\beta=109.513(6) \\
\gamma=93.035(7)\end{array}$ \\
\hline Volume $\left(\AA^{3}\right)$ & 2136.1(9) & $2463(2)$ \\
\hline $\mathrm{Z}$ & 2 & 2 \\
\hline Calculated density $\left(\mathrm{g} / \mathrm{cm}^{3}\right)$ & 1.308 & 1.272 \\
\hline Absorption coefficient $\left(\mathrm{mm}^{-1}\right)$ & 0.382 & 0.340 \\
\hline $\mathrm{F}(000)$ & 884 & 980 \\
\hline Crystal size $(\mathrm{mm})$ & $0.35 \times 0.34 \times 0.05$ & $0.2 \times 0.2 \times 0.2$ \\
\hline$\Theta$ range & 2.38 to $26.00^{\circ}$ & 2.255 to $25.500^{\circ}$ \\
\hline Limiting indices & $\begin{array}{l}-15 \leq h \leq 15 \\
-16 \leq k \leq 16 \\
-16 \leq l \leq 18\end{array}$ & $\begin{array}{l}-14 \leq h \leq 14 \\
-12 \leq k \leq 16 \\
-19 \leq l \leq 19\end{array}$ \\
\hline Reflections collected / unique & $\begin{array}{c}14376 / 8362 \\
{\left[R_{\text {int }}=0.0693\right]}\end{array}$ & $\begin{array}{c}16310 / 9053 \\
{\left[\mathrm{R}_{\mathrm{int}}=0.0612\right]}\end{array}$ \\
\hline Completeness to $\Theta$ & $99.4 \%$ & $98.8 \%$ \\
\hline Absorption correction & multi-scan ABSCOR & multi-scan ABSCOR \\
\hline Min. and max transmission & 0.731 and 1.000 & 0.851 and 1.000 \\
\hline Data / restraints / parameters & 8362 / 0 / 541 & $9053 / 0 / 622$ \\
\hline Goodness-of-fit on $\mathrm{F}^{2}$ & 1.010 & 1.042 \\
\hline $\begin{array}{l}\text { Final } R \text { indices } \\
{[I>2 \sigma(I)]}\end{array}$ & $\begin{array}{c}\mathrm{R}_{1}=0.0563 \\
\mathrm{wR}_{2}=0.1257\end{array}$ & $\begin{array}{c}\mathrm{R}_{1}=0.0539 \\
\mathrm{wR}_{2}=0.1270\end{array}$ \\
\hline $\mathrm{R}$ indices (all data) & $\begin{array}{c}\mathrm{R}_{1}=0.0685 \\
\mathrm{wR}_{2}=0.1352\end{array}$ & $\begin{array}{c}\mathrm{R}_{1}=0.0610 \\
\mathrm{wR}_{2}=0.1330\end{array}$ \\
\hline Largest diff. peak and hole $\left(\mathrm{e} \cdot \AA^{-3}\right)$ & 0.471 and -0.531 & 0.390 and -0.437 \\
\hline
\end{tabular}

${ }^{\S}$ Refinement method was full-matrix least-squares on $\mathrm{F}^{2}$; wavelength $=0.71073 \AA . \mathrm{R}_{1}=\sum|| F_{\mathrm{o}}|-| F_{\mathrm{c}}|| \sum\left|F_{\mathrm{o}}\right|$; $\mathrm{wR}_{2}=\left\{\sum\left[\mathrm{w}\left(F_{\mathrm{o}}^{2}-F_{\mathrm{c}}{ }^{2}\right)^{2}\right] / \sum\left[\mathrm{w}\left(F_{\mathrm{o}}{ }^{2}\right)^{2}\right]\right\}^{1 / 2}$. 